was scanned using a General Electric maxicamera II scanner and 400T formatter. $\quad 99 \mathrm{~m} T \mathrm{c}$ labelled diphosphonate was found to be concentrated within the plaques; activity being apparent by the sixth day.

To estimate the mean turnover rate of mineral in the plaques we are attempting to quantify this technique.

\section{CONCLUSIONS}

Simple calcergy is an easy, reproducible model of pathological calcification. Hydroxyapatite is found to deposit without forming intermediary crystalline phases and may nucleate on young collagen fibrils laid down during the inflammatory reaction. Mast cells are observed in large numbers during the initial phase of the inflammatory response, along with macrophages and fibroblasts. After 14 days fibroblasts are the predominant cell type, exhibiting electron microscopical profusions, and are actively laying down new collagen to encapsulate the affected area.

Isotope scanning may be a tool for assessing mineral turnover and for monitoring the progress of calcification.

The calcergy model is now being applied to investigate potential therapeutic inhibitors of hydroxyapatite deposition.

We would like to acknowledge the financial support of Ciba-Geigy Ltd.

\section{References}

1 Selye H, Tuchweber B, Gabbiani G.
Calcinosis induced by lead acetate. $J$ Pharmacol Exp Ther 1962; 128: 131.

2 Doyle D V, Dunn C T, Willoughby D A. Potassium permanganate induced calcergy: a model to study the effects of drugs on hydroxyapatite crystal deposition.J Pathol 1979; 128: 63.

3 Doyle D V. Tissue calcification and inflammation in osteoarthritis. J Pathol 1982; 136: 199-216.

4 McClure J. The effects of disodium ethane-hydroxy-1 1-diphosphonate and disodium dichloromethylene diphosphonate on lanthamide induced calcergy. J Pathol 1982; 137: 159-66.

5 McClure J. The effects of various anticalcific, anti-rheumatic and anti-inflammatory drugs on local (simple) calcergy induced by lead acetate in the mouse.J Pathol 1982; 137: 243-52.

6 Selye H, Ganniani G, Serafimou N. Histochemical studies on the role of the mast cell in calcergy. $J$ Histochem Cytochem 1964; 12: 563.

\title{
Periarticular calcification of the shoulder in articular chondrocalcinosis
}

\author{
JEAN C. GERSTER AND GEORGES RAPPOPORT
}

From the Rheumatology and Rehabilitation Centre and Radiology Department, University Hospital (CHUV), Lausanne, Switzerland

In articular chondrocalcinosis calcium deposits commonly occur within the articular cartilages and menisci; extra-articular linear deposits have, however, been described in the Achilles, quadriceps, triceps, and supraspinatus tendons. ${ }^{1-3}$

We report on the prevalence and morphology of periarticular calcification of the shoulder in patients suffering from articular chondrocalcinosis compared with a control group.

Two groups of patients were studied. The first comprised 30 consecutive patients ( 22 women, 8 men; mean age 73 years, range $40-89$ ) with definite articular chondrocalcinosis diagnosed radiologically in at least two joints. The disease was idiopathic in 28 cases; one was associated with hypothyroidism, and another with gout. A comparable control group ( 22 women, 8 men; mean age $72 \cdot 5$ years, range $41-90$ ) with no radiological evidence of articular chondrocalcinosis was taken at random and matched for sex and age. Patients with diabetes mellitus or severe renal insufficiency were not included in view of the increased prevalence of calcifying tendinitis among them. ${ }^{4}$

In all cases anteroposterior views of both shoulders were obtained with conventional radiological methods. When periarticular calcification was found xeroradiographic films were made to accentuate their density.

Periarticular calcification was found in nine patients with chondrocalcinosis; all had idiopathic disease; the calcium deposits were bilateral in three cases and unilateral in six. Calcification was also found in one control patient; the deposits were bilateral. The difference between the groups in those with calcification was significant $(p<0.006)$ by the exact Fisher test.

Morphologically, two types of calcification were encountered: linear punctate and dense homogeneous. Linear punctate calcification was found in eight patients in the study group; in two cases punctate calcium deposits were also observed at the insertion of the long head of the biceps. Dense homogeneous calcification was found in one control and four of the study group, in three of whom they were associated with linear calcification. The difference between the groups in prevalence of dense calcification is, however, not significant $(\mathrm{p}=0 \cdot 18)$.

Of the 10 cases with periarticular calcification, three (all in the study group) had chronic shoulder pain, one suffering from a rupture of the rotator cuff; the remainder were asymptomatic. 
These results show that periarticular calcification of the shoulder, often clinically silent, is common among patients with articular chondrocalcinosis. It is found mainly at the insertion of the supraspinatus tendon.

Eight patients in the study group had fine linear tendon calcification. This calcification is supposed to be made of calcium pyrophosphate dihydrate crystal deposits, inasmuch as their morphology is similar to that observed in other tendons with typical CPPD crystal deposits. ${ }^{23}$ However, the prevalence in the supraspinatus tendon is twice that in the Achilles or quadriceps tendons ${ }^{2}$; this could be because of repeated strains due to the great mobility of the shoulder joints.

Dense homogeneous tendon calcification may be considered to result from apatite deposits, ${ }^{12}$ which are the most common cause of calcifying tendinitis of the shoulder. This was more frequent, but not significantly so, in the study group than in the control group; further radiological studies are needed to see whether apatite deposition disease could be associated with articular chondrocalcinosis. In three cases both linear and dense tendon deposits were present; these findings have to be compared with those of a triceps tendon where both CPPD and hydroxyapatite crystals could be demonstrated by radiocrystallography, ${ }^{3}$ suggesting that mixed crystal deposition disease may occur in certain tendons. There is an analogy with what occurs in some osteoarthritic joints where the two kinds of crystals can coexist. ${ }^{5}$

\section{References}

1 Genant H K. Roentgenographic aspects of calcium pyrophosphate dihydrate crystal deposition disease (pseudogout). Arthritis Rheum 1976; 19: 307-28.

2 Gerster J C, Baud C A, Lagier R, Boussina I, Fallet $\mathrm{G} \mathrm{H}$. Tendon calcifications in chondrocalcinosis. A clinical, radiologic, histologic and crystallographic study. Arthritis Rheum 1977; 20: 717-22.

3 Gerster J C, Lagier R, Boivin G. Olecranon bursitis related to calcium pyrophosphate dihydrate crystal deposition disease. Clinical and pathologic study. Arthritis Rheum 1982; 25: 989-96.

4 Resnick D. Calcium hydroxyapatite crystal deposition disease. In: Resnick D, Niwayama G, eds. Diagnosis of bone and joint disorders. Philadelphia, London, Toronto: W B Saunders, 1981: 1575-97.

5 Dieppe P A, Doyle D V, Huskisson E C, Willoughby D A, Crocker P R. Mixed crystal deposition disease and osteoarthritis. Br Med J 1978; i: 150.

\title{
Articular chondrocalcinosis, quadriceps calcification, and patellofemoral degeneration in the elderly
}

\author{
EDWARD WILKINS, AND GORDONEVISON
}

From St Martin's Hospital, Bath

Linear calcific deposits in the quadriceps tendon ${ }^{2}$ and 'isolated' patellofemoral degeneration ${ }^{34}$ have been described as radiographic features seen in association with intra-articular chondrocalcinosis. It has been suggested that their presence may be of value in distinguishing between pyrophosphate arthropathy and other forms of degenerative joint disease.

We assessed the influence of aging on this interrelationship by analysing radiographs taken on consecutive admissions to an acute geriatric unit. We documented the prevalence of intra-articular chondrocalcinosis, patellofemoral degeneration, and quadriceps calcification and defined their interrelationship.

METHODS AND RESULTS

A total of 120 consecutive $x$-ray films of the knee (anteroposterior and lateral), pelvis, and wrists were analysed by four independent observers, using light intensification and magnification. Articular chondrocalcinosis was defined as the finding of dense, hazy, linear, or stippled intra-articular calcification. Quadriceps calcification as seen on lateral views of the knee was defined as either in the muscle belly or in the tendinous insertion. Formation of osteophyte on the upper margin of the articular surface of the patella, loss of articular cartilage in the patellofemoral joint, and subchondral sclerosis was used to assess the presence of patellofemoral degeneration, classified as mild, moderate, or severe.

To achieve maximum sensitivity and to avoid excessive radiation exposure, Ilford rapid $\mathbf{R}$ film was used for the knee $x$-ray examinations, and $\mathrm{X}-6-\mathrm{Mat} \mathrm{RP}-\mathrm{X}-\mathrm{RPI}$ for the pelvis.

Data were avilable on 100 patients (31 men, 69 women) aged from 65 to 97 years (mean (SD) $79 \cdot 4$ years $(6 \cdot 6)$ ). None of the patients had haemochromatosis or hypercalcaemia.

Thirty-four patients had intra-articular chondrocalcinosis; 25 had changes at the knee. The prevalence rose from $15 \%$ ( 3 out of 20 ) in those aged $65-74$ to $36 \%$ (20 out of 55) in those aged 75-84, and to $44 \%$ ( 11 out of 25 ) in patients aged from 85 to 97 years.

Quadriceps calcification at the tendinous insertion was seen in 54 patients. The prevalence increased from $20 \%$ in patients aged 65 to 74 years to $60 \%$ in those aged 75 to 84 , to $64 \%$ in those aged 84 to 97 .

Fifty patients had evidence of patellofemoral degeneration. In 13 patients the changes were mild, in 27 patients moderate, and in 10 severe. In 17 cases radiographs showed no other abnormality - that is, degeneration was isolated. The prevalence rose from $35 \%$ in patients aged 65 to 74 to $49 \%$ in those aged 75 to 84 years, and to $64 \%$ in patients above 84 years.

The table shows the 